\title{
Outcomes Analyses of the Outpatient Treatment of Venous Thromboembolic Disease Using the Low-Molecular-Weight Heparin Enoxaparin in a Managed Care Organization
}

\begin{abstract}
OBJECTIVE: To analyze the implementation and feasibility of an outpatientbased treatment protocol for uncomplicated deep venous thrombosis (DVT) using the low-molecular-weight heparin (LMWH) enoxaparin in a managed care setting by measuring utilization and outcomes data and pharmacoeconomic indicators.
\end{abstract}

DESIGN: Prospective cohort study of outpatients treated using the LMWH enoxaparin in a clinical pharmacist-managed anticoagulation clinic (AC) during an 18month period. Retrospective analysis compared these patients to an inpatient group that had received intravenous unfractionated heparin (IVUH) for DVT treatment the previous year.

SETTING: Lovelace Health Systems, an integrated health care delivery system with a staff-model HMO.

MAIN OUTCOME INDICATORS: Utilization demographics, short-term clinical outcomes, patient/provider satisfaction indices, and institution-wide costs.

RESULTS: $61 \%$ of all patients with DVT were treated as outpatients, with $42 \%$ treated completely at home. There were no significant differences in the incidence of primary adverse outcomes or secondary adverse outcomes between the LMWH-treated group treated as outpatients $(n=102)$ and the previous year's comorbid-matched IVUH-treated group treated in-hospital $(n=97)$. There were no significant differences in the rates of thrombocytopenia or minor bleeding events. The LMWH-treated group reported very-good-to-excellent patient/provider satisfaction indices. There was an average institution-wide cost savings of $\$ 2,473$ per patient when prefilled enoxaparin syringes were used.

CONCLUSION: With appropriate exclusionary criteria and risk stratification strategies and using a pharmacistmanaged anticoagulation service with home health service support, an outpatient-based treatment protocol for DVT using the LMWH enoxaparin results in comparable short-term clinical adverse outcomes when compared with inpatient treatment using IVUH, is associated with high patient and provider satisfaction indices, and is cost-effective in a managed care setting.

KEYWORDS: venous thromboembolic disease, outpatient DVT treatment, pharmacist-managed anticoagulation service

J Managed Care Pharm 2000: 298-304 by Alex C. Spyropoulos, Joe Kardos, and Pam Wigal

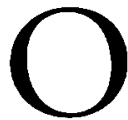

utpatient-based treatment protocols using lowmolecular-weight heparin (LMWH) for the management of deep venous thrombosis (DVT) are well established in a clinical trial setting. .-8 $^{-8}$ The safety, efficacy, feasibility, patient/physician satisfaction, and potential cost savings when these protocols are translated to a routine care setting are less well established. Outcome analyses of DVT home treatment programs are rare; they reflect heterogeneous patient selection, implementation, safety, efficacy, and cost savings..$^{9-12}$ Indeed, only recently has the Food and Drug Administration approved one LMWH (enoxaparin) for the outpatient treatment of lower extremity DVT. Given that home treatment of DVT will greatly expand in coming years, data to reflect appropriate patient selection and risk stratification criteria, patient and provider satisfaction indices, and institution-wide cost savings in a routine care setting would enable more effective use of LMWH on an outpatient basis.

\section{Methods}

\section{Study Design}

A prospective cohort study of 167 consecutive patients with DVT referred to our institution's pharmacist-managed anticoagulation clinic (AC) from March 1997 to August 1998 was conducted. The study group that was treated on an outpatient basis was divided into an outpatient group (OP) that did not meet exclusionary criteria and was treated on a completely outpatient basis, and an observation group (OBS) that was treated with a reduced hospital stay because of physician discomfort with outpatient treatment or the need to further risk-stratify the patient (refer to outpatient treatment protocol).

Clinical and economic outcome indicators were then compared to those of a previous year's inpatient group (IP) that received intravenous unfractionated heparin (IVUH) in-hospital for DVT. The 1.996 group had the diagnosis of DVT established with duplex ultrasonography and received warfarin concomitantly with IVUH.

\section{Outpatient Treatment Protocol}

Our institution, an integrated health maintenance organization, developed an outpatient-based treatment protocol using 


\section{Outcomes Analyses of the Outpatient Treatment of Venous Thromboembolic Disease Using the Low-Molecular-Weight Heparin Enoxaparin in a Managed Care Organization}

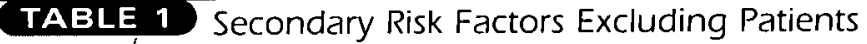 from Outpatient-Based Treatment Protocol for Deep Venous Thrombosis with Low-Molecular-Weight Heparin}

- Plateles count lower than $100 \times 10^{9} / \mathrm{L}$

- Aclive hemorrhage

- Hislory of gastrointestinal bleeding within six months

- Underlying liver disorder (INR>1.5)

- History of familial bleeding disorder

- Hypertensive urgency or emergency (SBP>220 and DBP>120)

- Congenital or acquired hypercoagulable state

- Catheter-associated deep venous thrombosis

- History of heparin sensitivity

- Pregnancy or lactating lemale

- Pulmonary embolism ${ }^{\mathrm{a}, \mathrm{b}}$

- Iliofemoral thrombosis

- Morbid obesity (>30\% IBW $)^{\mathrm{a}, \mathrm{c}}$

- Renal insufficiency (serum creatinine>2.5)

- Recent surgery (especially intraabdominal or intracranial)

- Other comorbid medical conditionsa

- Other factors increasing risk of home treatment (compliance, insurance issues)

Notes: Low Risk=no risk factors. High Risk=one or more absolute risk factors or multiple relative risk factors. Moderate Risk=one relative risk factor. ${ }^{a}$ Relative exclusionary risk factors.

Maximum of $150 \mathrm{~kg}$.

'Clinically stable pulmonary embolism (nemodynamically stable, no evidence of right ventricular strain by echocardiogram, no significant hypoxia, $<30 \%$ ventilation/perfusion mismatch by lung scan, minimal symptoms). INR is International Normalized Ratio. IBW is ideal body weight.

enoxaparin for uncomplicated proximal lower extremity DVT under the Episodes of Care Disease Management Program that utilized a multidisciplinary team of physicians, nurses, pharmacists, social workers, and data management personnel. The diagnosis of proximal DVT was established by duplex ultrasonography, and the diagnosis of pulmonary embolism (PE) was established by ventilation/perfusion scan. Three treatment alternatives were developed based on time of day, patient reliability, physician comfort level, and a set of absolute and relative exclusionary criteria that precluded home treatment (see Table 1). These treatment alternatives consisted of:

- a completely outpatient-based protocol (OP) using enoxaparin in those patients with a low risk profile who did not meet exclusionary criteria;

- an inpatient protocol (IP) using a weight-based nomogram for intravenous unfractionated heparin in those patients with a high-risk profile who met one or more exclusionary criteria; and

- a protocol that necessitated an abbreviated hospitalization
(OBS) using enoxaparin (usually less than 24 hours) followed by outpatient treatment in those patients with a moderate risk profile based on comorbidity, home treatment issues, and other factors but who did not meet absolute exclusionary criteria, or patients whose risk profile, compliance, insurance reimbursement status, etc., was unknown at the time of treatment but who were felt to be acceptable outpatient candidates by the treating physician (Figure 1 ).

For those patients who qualified as outpatients, a complete history was performed to rule out thrombophilic states, bleeding tendencies, and recent surgical procedures, and a thorough physical exam was performed that included pulse oximetry readings and stool for occult blood. Baseline laboratory measurements included hemoglobin, platelet count, creatinine, liver function studies, PT, a PTT, and urinalysis.

If the patient continued not to meet exclusionary criteria after careful assessment of the history, physical, and initial laboratory analysis, LMWH therapy was initiated. Enoxaparin was given at a dose of $1 \mathrm{mg} / \mathrm{kg}$ SQ Q 12 hours with a maximum dose of $150 \mathrm{mg}$ Q12 hours. Concomitantly with enoxaparin, all patients were started on warfarin at a dose of 5-10 mg; enoxaparin was discontinued after at least a five-day overlap with warfarin and a target international normalization ratio (INR) of 2.0 to 3.0 was achieved on two successive days of therapy.

All potential outpatients were evaluated in the $A C$ for their ability to self-inject. A patient who was successfully evaluated received an education packet that contained enoxaparin and warfarin instructions. When appropriate, patients, including those patients unable to self-inject, were also enrolled in Home Health Care Services.

All outpatients were monitored by the AC and were treated for a minimum of 90 days, at which time they completed the satisfaction questionnaire. In addition, all patients who completed their DVT treatment in-hospital were also enrolled in the AC. The health network physicians also received a satisfaction questionnaire related to the $\mathrm{AC}$ in conjunction with the outpatient DVT protocols.

The protocols went into effect in March 1997, when a fulltime pharmacist-managed $\mathrm{AC}$ was established with a dedicated medical director who acquired expertise in anticoagulation management. A dedicated Home Health Service was also instituted to facilitate home discharge and outpatient treatment. In addition, through our institution's Disease Management Program, data analysis and support services were instituted for the AC. Initially, the protocols were used by a small group of physicians who became well acquainted with them; they included the Anticoagulation Group physicians, our institution's Hospitalist Team, and physicians associated with the Vascular Lab. After an extensive educational campaign, the protocols were gradually expanded to the rest of the physician group over six months. 
Outcomes Analyses of the Outpatient Treatment of Venous Thromboembolic Disease

Using the Low-Molecular-Weight Heparin Enoxaparin in a Managed Care Organization

\section{FIGURE 1 Lovelace Health Systems Outpatient Protocol for Treatment of}

Deep Venous Thrombosis Using Low-Molecular-Weight Heparin

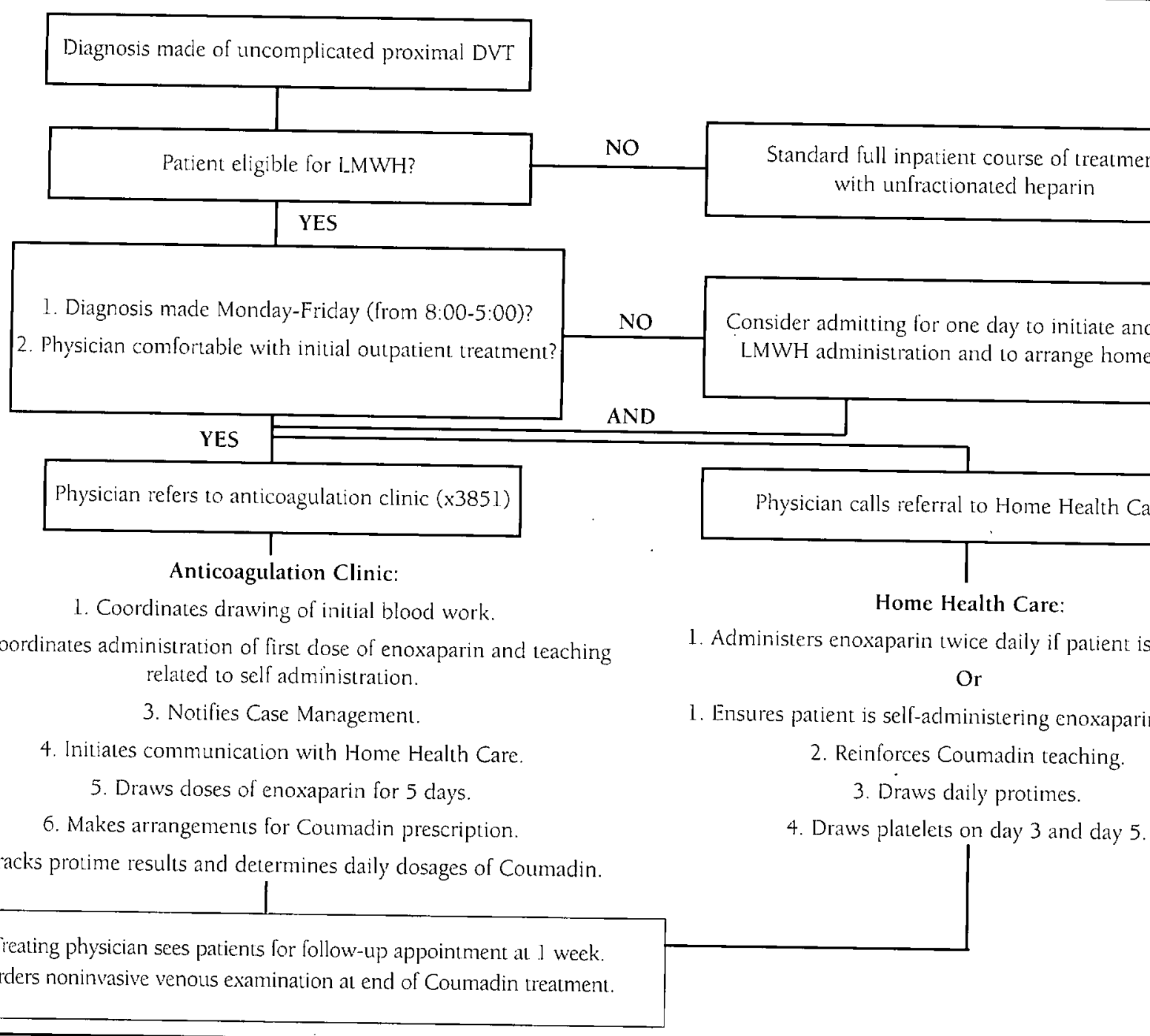

Notes: DVT is decp venous thrombosis. LMWH is low-molecular-weight heparin

\section{Data Analysis}

Outcome data, including utilization demographics, primary and secondary clinical adverse outcomes, and satisfaction survey results, were monitored for patients enrolled in the outpatient DVT treatment program through the AC $(n=1.02)$. This analysis was then compared retrospectively to a 1996 comorbid matched inpatient group $(n=97)$. Pharmacoeconomic analysis captured 15 more hospitalized venous thromboembolism patients in 1996 who were treated with IVUH.

Primary clinical adverse outcomes were defined as symptomatic recurrent DVT, PE, clot propagation at the end of heparin therapy, or death during treatment. Secondary clinical adverse outcomes were defined as major bleeding (drop in hemoglobin of $>2.0 \mathrm{~g} / \mathrm{dl}$ or need for transfusion of two or more units packed red blood cells), intracranial bleeding, or retroperitoneal bleeding. Significant thrombocytopenia was defined as a platelet count less than $100 \times 10^{9} /$ L with or without heparin-associated antiplatelet antibodies. Minor bleeding episodes were defined as epistaxis, ecchymoses, hematuria, and other bleeding (ICD-9 459.0). All clinical outcomes were captured within 90 days of initiation of treatment. Frequency of adverse outcomes for the two cohort groups was analyzed by a two-sample t-lest assum- 


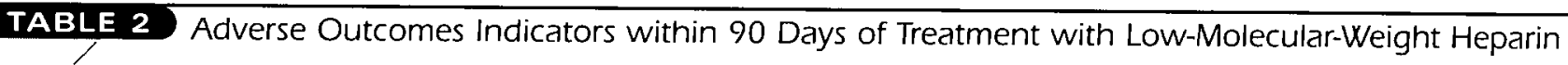

\begin{tabular}{|c|c|c|c|c|}
\hline \multirow[b]{3}{*}{ Primary adverse outcomes } & \multicolumn{2}{|c|}{ Enoxaparin $(n=102)^{a}$} & \multirow{2}{*}{$\begin{array}{l}\text { IVUH }(\mathrm{n}=97)^{\mathrm{b}} \\
\text { Inpatient Group }\end{array}$} & \multirow[t]{2}{*}{ Significance } \\
\hline & Outpatient Group & Observation Group & & \\
\hline & & & & \\
\hline Recurrent deep venous thrombosis & 0 & 0 & 2 & \\
\hline Clot propagation & 1 & 0 & 0 & \\
\hline Pulmonary embolism & 0 & 1 & 2 & \\
\hline Death from thromboembolism & 0 & 0 & 0 & \\
\hline Total & \multicolumn{2}{|c|}{$2(1.9 \%)$} & $4(4.1 \%)$ & $p=.38$ \\
\hline \multicolumn{5}{|l|}{ Secondary adverse outcomes } \\
\hline Major bleeding & 0 & 0 & 0 & \\
\hline Intracranial bleeding & 0 & 0 & 0 & \\
\hline Retroperitoneal bleeding & 0 & 0 & 0 & \\
\hline Total & \multicolumn{2}{|c|}{$0(0 \%)$} & $0(0 \%)$ & $p>.99$ \\
\hline Thrombocytopenia & 0 & 0 & 0 & \\
\hline Total & \multicolumn{2}{|c|}{$0(0 \%)$} & $0(0 \%)$ & $p>.99$ \\
\hline \multicolumn{5}{|l|}{ Minor adverse outcomes } \\
\hline Minor bleeding/bruising & 1 & 0 & 1 & \\
\hline Increased symptomatology & 2 & 0 & 0 & $\cdot$ \\
\hline Total & \multicolumn{2}{|c|}{$3(2.9 \%)$} & $1(1.0 \%)$ & $p=34$ \\
\hline
\end{tabular}

Note: IVUH is intravenous unfractionated heparin.

"March 1997-August 1998

${ }^{b} 1996$ (primary diagnosis of deep venous thrombosis without exclusionary criteria).

ing equal variances, for a difference in means at the $95 \%$ confidence interval.

The patient satisfaction survey had seven questions answered on a five-point scale plus a comment section. The provider satisfaction survey had six questions answered on a two- to four-point scale plus a comment section.

The pharmacoeconomic data captured costs or, in the few instances where actual costs could not be calculated, cost-tocharge ratios. Costs for standard inpatient treatment with IVUH included personnel (MD, nurse, educator, home health services, and lab personnel), drugs and supplies (UH premix bag, vial for bolus, IV and IV tubing), lab testing (PTT test kit), and hospital (room maintenance, laundry and housekeeping, nursing, etc.). Costs for outpatient DVT treatment program included personnel (MD, nurse, nurse educator, home health services, and anticoagulation clinic services), drugs and supplies (enoxaparin at $\$ 11.92$ per $30 \mathrm{mg}$ syringe, sharps container, and filling of syringes by pharmacy personnel), hospital costs, and lab testing.

After the advent of prefilled enoxaparin syringes, drug costs for the LMWH-treated group were adjusted to reflect the fact that pharmacy personnel no longer needed to fill syringes. Chronic warfarin administration and monitoring during the maintenance phase of therapy were not included in the cost analysis. The start-up costs for the AC, including office space, computers, and pharmacy personnel, were not included in the outpatient cost determination because they were already in place when the DVT program began.

\section{Results}

From March 1997-August 1998, 61\% (102/167) of all patients with DVT were treated as outpatients. Of these, 69\% (70/102) had a low risk profile and were treated on a completely outpatient basis. The remaining 65 patients who met exclusionary criteria were treated in-hospital with intravenous heparin (50 patients) or were oncology patients who were treated off-protocol with LMWH (1.5 patients).

Of the 102 patients who completed outpatient treatment, 93 (91\%) had uncomplicated lower extremity DVT, 6(6\%) had concurrent clinically stable PE, and $3(3 \%)$ had upper extremity DVT that was not catheter-associated (spontaneous or effort- 


\section{Outcomes Analyses of the Outpatient Treatment of Venous Thromboembolic Disease Using the Low-Molecular-Weight Heparin Enoxaparin in a Managed Care Organization}

\section{TABLE 3 Lovelace Health Systems Anticoagulation Episodes of Care: 1996 Inpatient vs. 1997-98 Projected and Actual Costs for DVT Protocol}

Expense Category
Annual Comparison" IP Actual Protocol with Standard Heparin (1996)
1/96 through 1.2/96

Projected 12-month Expenses

Protocol with LMWH
3/97 through 8/98

Actual 18-month

Expenses Protocol with LMWH

\begin{tabular}{|c|c|c|c|}
\hline Utilization statistics & & & \\
\hline Annual number of patients & 112 & 93 & 102 \\
\hline Annual number of patient days & 594 & 54 & 81 \\
\hline Average length of stay (days) & 5.30 & 0.58 & 0.79 \\
\hline Annual number of visits by home health providers & 560 & 339 & 527 \\
\hline Variable expense categories & & & \\
\hline Personnel (MD, Rx, Education, Lab) & $\$ 1.28,694$ & $\$ 71,664$ & $\$ 75,883$ \\
\hline Room maintenance (laundry, housekeeping, etc.) & 322,278 & 28,226 & 46,946 \\
\hline Drugs and supplies & 20,295 & 78,942 & 93,099 \\
\hline Lab testing & 13,021 & 25,234 & 13,342 \\
\hline Total expenses & $\$ 484,288$ & $\$ 204,066$ & $\$ 229,270$ \\
\hline Per patient case expenses & $\$ 4,324$ & $\$ 2,1.94$ & $\$ 2,248(1,851)^{\mathrm{h}}$ \\
\hline Potential and actual expense reductions & & $\$ 198,066$ & $\$ 211,778$ \\
\hline
\end{tabular}

Notes: DVT is deep venous thrombosis. IP is inpatient. LMWH is low-molecular-weight heparin. Costs were compiled based on actual costs and cost-tocharge ratios.

"Comparison group is based on 1996 patients with a primary diagnosis of thrombosis $(n=112)$

${ }^{b}$ Reflects decrease in per patient case expenses after advent of prefilled enoxaparin syringes, which decreased pharmacy personnel costs for filling syringes.

related). The average age of the patients treated at home was 61.7 years; the average age of females was 63.9 years and of males, 59.6 years.

After 90 days of anticoagulant therapy, adverse outcomes data revealed no significant difference for a primary adverse outcome event between the OP or OBS group treated with LMWH and the IP group treated with IVUH, although the IP group had double the incidence of primary adverse outcome events (2/1.02 [1.9\%] versus $4 / 97[4.1 \%], p=38$ ). (In the LMWH-treated group one patient had a PE and one patient had clot propagation, while the IP group had two patients with PE and two patients with recurrent DVT.) There were no major episodes of bleeding or significant thrombocytopenia in either the LMWH-treated group or inpatient group. The incidence of minor bleeding and increased symptomatology at the injection site was slightly higher in the LMWH-treated group than in the inpatient group, but the difference was not significant (3/102 $[2.9 \%$ ] versus $1 / 97$ [1.0\%], $p=34$ ). A two-sample $t$-test assuming equal variances for a difference in means at the $95 \%$ confidence interval revealed no significant difference between study groups. The short-term clinical adverse outcome data are summarized in Table 2

The patient satisfaction question that measured overall experience with the $A C$ and outpatient DVT program revealed that $41 / 49(84 \%)$ of respondants had a very-good-to-excellent experience; the average rating was 4.10 (1=poor, $5=$ excellent). In the comments on the questionnaire, avoidance of hospital stay, less disruption in routine, quick return to work, and thorough follow-up by the AC constituted the majority of reasons for patient satisfaction. There was no mention of injection issues as being problems.

In terms of provider satisfaction, 38/57 (67\%) of providers responded that the AC greatly improved quality of care. The comment section cited more efficient monitoring and time management as the major reasons, especially with the outpatient DVT protocols.

Economic analysis revealed that the annual number of patient days in-hospital was reduced from 594 to 81 days, comparing by retrospective analysis the IP group from 1996 to the outpatient LMWH-treated group during the study period. The 


\section{Outcomes Analyses of the Outpatient Treatment of Venous Thromboembolic Disease Using the Low-Molecular-Weight Heparin Enoxaparin in a Managed Care Organization}

average hospital stay for DVT treatment was reduced from 5.3 days to 0.79 days. Variable expense categories including personnel fees (physician, anticoagulation clinic, etc.), and hospital bed costs revealed significant cost reductions for the outpatient group ( $-\$ 52,811$ for personnel and $-\$ 275,332$ for bed costs), although the costs of their drugs and supplies $(+\$ 72,804)$ and lab testing $(+\$ 321)$ were greater. Per-patient case expense totaled $\$ 4,324$ for the IP group and $\$ 2,248$ for the LMWH-treated group, for an initial institution-wide total cost savings of $\$ 211,778$, or a per-patient average cost savings of $\$ 2,076$. Cost savings improved to $\$ 2,473$ per patient treated after the advent of prefilled syringes decreased pharmacy personnel costs. These results are summarized in Table 3

\section{Discussion}

These data represent one of the larger clinical experiences in the United States in a routine care setting for the outpatient treatment of VTE using LMWH. With careful patient selection and risk stratification criteria, approximately $61 \%$ of all VTE patients could be treated as outpatients. This eligibility figure is more conservative than some previous outpatient figures for a hospital-in-the-home DVT treatment program (79\% and $83 \%)^{10,12}$ Furthermore, $42 \%$ of the total DVT patient population in our study met a low-risk profile and were treated completely as outpatients, which coincides well with figures of $49 \%$ and $36 \%$ seen in previous randomized clinical trials. ${ }^{3-+}$

Although this study used only a small sample, the DVT recurrence rate of $1.9 \%$ and major bleed rate of $0 \%$ are among the lowest reported to date for an outpatient treatment program; they compare favorably both to published reports and to our institution's previous inpatient experience. ${ }^{2-10}$ Strict patient exclusionary criteria that correspond well to a previous report on selection criteria for outpatient management of proximal lower extremity DVT, plus a more formal risk stratification strategy, may well explain the low number of adverse outcomes. ${ }^{13}$ In addition, the benefits of a pharmacist-managed $A C$ in terms of improved anticoagulation control and reduced thromboembolic and bleeding rates may also have contributed to the lower frequency of clinical adverse outcomes. ${ }^{14}$

As seen in previous studies, the outpatient treatment program was associated with high patient satisfaction. ${ }^{+.8 .10}$ Due to extensive home health services, injection issues did not play a significant role in patient perception of home treatment. The home treatment program and the AC were also associated with high provider satisfaction, especially with regard to patient monitoring.

The institution-wide cost savings of $\$ 2,473$ per patient treated with the prefilled enoxaparin syringes is significant. It compares favorably with other pharmacoeconomic reports of established or projected cost savings to a health care system using LMWH therapy as compared with IVUH therapy in DVT treatment, especially in the outpatient setting. ${ }^{8.11,15-17}$ Most of the cost savings was due to reduced hospital stays, although improved patient monitoring by the AC may also have played a role. Indeed, the cost savings figure is likely an underestimate, because today's hospital costs are higher than 1.996 costs.

Successfully implementing an outpatient-based DVT treatment program using LMWH in a routine care setting requires three major determinants: protocols that have strict patient selection and risk stratification criteria; a dedicated AC with home health service support; and a single physician or a small group of physicians who have expertise in anticoagulation management. A data analysis system would also be of benefit, especially for tracking adverse and cost-benefit outcomes.

Patients with a low risk-factor profile may be considered for treatment on a completely outpatient basis with home health service support if injection problems arise. High-risk patients should still be treated in a hospital (whether or not to use LMWH will depend on the institution's cost analysis for IVUH). Patients with a moderate risk profile, or one that cannot be determined at time of treatment, and those whose compliance or insurance assessment with regard to LMWH is questionable may benefit from a short hospital stay. Completing treatment as an outpatient may be considered if these issues are resolved. This group of patients will usually benefit from home health service support.

Whether to extend outpatient DVT protocols to include other forms of VTE remains controversial. Some studies support initiating therapy of hemodynamically stable PE with LMWH as a safe and efficacious alternative to therapy with IVUH, but whether this can routinely be done on an outpatient basis needs to be elucidated with additional randomized, controlled trials., ${ }^{5,11}$ Upper extremity DVT that is not catheter-associated (i.e., PagetSchroetter syndrome) in otherwise healthy patients may be well suited for management with $\mathrm{LMWH}$ in an outpatient setting.

\section{References}

1. Hull RD, Raskob GE, Pinea GF, et al. Subcutaneous low-molecular-weightheparin compared with continuous intravenous heparin in the treatment of proximal-vein thrombosis. N Engl J Med1992; 326: 975-82.

2. Lensing AWA, Prins MH, Davidson BL, Hirsch J. Treatment of deep venous thrombosis with low-molecular-weight heparins: a meta-analysis. Arch Intern Med 1995; 155: 60 J-07.

3. Levine M, Gent M, Hirsh J, et al. A comparison of low-molecular-weightheparin administered primarily at home with unfractionated heparin administered in the hospital for proximal deep-vein thrombosis. N Engl J Med 1996; 334: 677-81.

4. Koopman MMW, Prandoni P, Piovella F, et al. Treatment of venous thrombosis with intravenous unfractionated heparin administered in the hospital as compared with subcutaneous low-molecular-weight heparin administered at home. N Engl J Med 1996; 334: 682-87.

5. Simonneau $G$, Sors $H$, Charbonnier B, et al. A comparison of low-molecular weightheparin with unfractionated heparin for acute pulmonary embolism. $N$ Engl J Med 1997; 337: 663-69.

6. The Columbus Investigators. Low-molecular-weight heparin in the treat- 


\section{Outcomes Analyses of the Outpatient Treatment of Venous Thromboembolic Disease Using the Low-Molecular-Weight Heparin Enoxaparin in a Managed Care Organization}

ment of patients with venous thromboembolism. N Engl J Med 1997; 337: $657-62$

7. Turkstra F, Koopman MM, Buller HR. The treatment of deep venous thrombosis and pulmonary embolism. Thromb Heamost 1997; 78: 489-96

8. Goldhaber SZ, Morrison RB, Diran LL, et al. Abbreviated hospilalization for deep venous thrombosis with the use of ardreparin. Arch Intern Med 1998; 158: $2325-28$.

9. Dedden $P$, Chang B, Nagel D. Pharmacy-managed program for home treatment of deep vein thrombosis with enoxaparin. Am J Health Syst Pharm 1997; 54: 1968-72.

10. Hanison L, McGinnis J, Crowher M, et al. Assessment of outpatient treatment of deep-vein thrombosis with low-molecular-weight heparin. Arch Intern Med 1998; 1.58: 2001-03.

11. Ting SB, Ziegenbein RW, Gan TE, et al. Dalteparin for deep venous thrombosis: a hospital-in-the-home program. Med J Aust 1998; 168: 272-76.

12. Wells PS, Kovacs MJ, Bormanis J, et al. Expanding eligibility for outpatient treatment of deep venous thrombosis and pulmonary embolism with lowmolecular-weight heparin. Arch Intern Med 1998; 158: 1809-12.

13. Yusen RD, Haraden BM, Gage BF, et al. Criteria for outpatient management of proximal lower extremity deep venous thrombosis. Chest $1999 ; 115$ : 972-79

14. Chiquette E, Amato MG, Bussey HI. Comparison of an anticoagulation clinic with usual medical care. Arch Intern Med 1998; 158: 1641-47.

15. Hull RD, Raskob GE, Rosenbloom D, el al. Treatment of proximal vein thrombosis with subcutaneous low-molecular-weight heparin vs intravenous heparin. Arch intern Med 1997: 157: 289-94.

16. O'Brien B, Levine M, Willan A, et al. Economic evaluation of outpatient treatment with low-molecular-weight heparin for proximal venous thrombosis. Arch Intern Med 1999; 159: 2298-04

17. Gould MK, Dembitzer AD, Sanders GD, et al. Low-molecular-weight heparins compared with unfractionated heparin for treatment of acute deep venous thrombosis. A cost-effectiveness analysis. Ann Intern Med 1999; 130: 789-99. 